\title{
Section Showing Minimal Intra-individual Variations in Masticatory Movement
}

\author{
Hiroshi Shiga, BEng, DDS, PhD, ${ }^{a}$ Yoshinori Kobayashi, DDS, PhD, ${ }^{a}$ \\ Christian S. Stohler, DMD, DrMedDent, ${ }^{\mathrm{b}}$ and Akira Tanaka, DDS, PhDa \\ a Department of Partial and Complete Denture, School of Life Dentistry at Tokyo, The Nippon Dental University, \\ Tokyo, Japan \\ b School of Dentistry, University of Maryland, Baltimore, MD, USA
}

\section{Clinical significance}

This study shows that the masticatory movement of the mandibular incisal point during mastication of softened chewing gum remains almost constant over about ten cycles after the initial few cycles.

\begin{abstract}
Abstarct
Purpose: To clarify the section showing minimal intraindividual variations in the movement of the mandibular incisal point during mastication of softened chewing gum.

Methods: Twenty healthy subjects were asked to chew softened chewing gum on the habitual side for $20 \mathrm{sec}^{-}$ onds. The change in the spatial parameters (gape and masticatory width) and temporal parameter (cycle time) were investigated for 20 cycles from the first cycle. The coefficients of variation of these parameters were investigated for each of 10 consecutive cycles (first to eleventh series).

Results: The spatial and temporal parameters were maximal at the first cycle, decreased progressively until the fourth or fifth cycle, and then remained almost unchanged thereafter. The coefficients of variation of the parameters were maximal during the first series, decreased progressively until the fourth to sixth series, and then tended to increase gradually thereafter. Minimal coefficients of variation were observed during the fifth and sixth series for the gape, during the fifth series for the width, and during the fourth series for the cycle time.

Conclusion: These results suggest that the ten cycles after the fourth to the sixth cycle was the section showing minimal intra-individual variations in the masticatory movement during the chewing of softened chewing gum.
\end{abstract}

\section{Corresponding to: Dr Hiroshi Shiga}

Department of Partial and Complete Denture,

School of Life Dentistry at Tokyo, The Nippon Dental

University

1-9-20 Fujimi, Chiyoda-ku, Tokyo 102-8159, Japan

Tel: +81-3-3261-5729, Fax: +81-3-3261-8464

E-mail: h-shiga@tokyo.ndu.ac.jp

Received on August 10, 2007/Accepted on November 26, 2007
Key words: mastication, intra-individual variation, gape, masticatory width, cycle time

\section{Introduction}

During mastication, a rhythmical pattern is maintained by the pattern generator in the brain stem. ${ }^{1}$ Regulation of this movement is also derived on the basis of feedback information from the periphery, such as the teeth, masticatory muscles, and temporomandibular joints..$^{2-5}$ Therefore, objective evaluation of mastication might be helpful in the evaluation or diagnosis of disorders of the peripheral structures, including functional problems related to these structures. Based on such expectations, analyses of the spatial and temporal parameters during mastication have been attempted which demonstrate that while healthy subjects show regular and stable movements, subjects with malocclusion or temporomandibular disorders exhibit irregular and unstable movements. ${ }^{6-10}$

Mastication may be affected both by exogenous $\mathrm{s}^{3-5,11}$ and endogenous factors, ${ }^{7,8,12-16}$ and can vary even among healthy subjects. Therefore, when evaluating mastication, it is necessary to minimize the inter-individual and intra-individual variations arising from these factors. We previously reported that when comparing the masticatory movement of free side chewing with unilateral chewing during mastication of foods with varying properties, the inter-individual variations were minimal during mastication of softened chewing gum on the habitual side. ${ }^{17}$ Although intra-individual variations in the stability of masticatory movement have been examined, ${ }^{18}$ intra-individual variations in the masticatory path, such as the gape and masticatory width, have never been evaluated.

Therefore this study was undertaken to clarify the section in masticatory movement in which the 


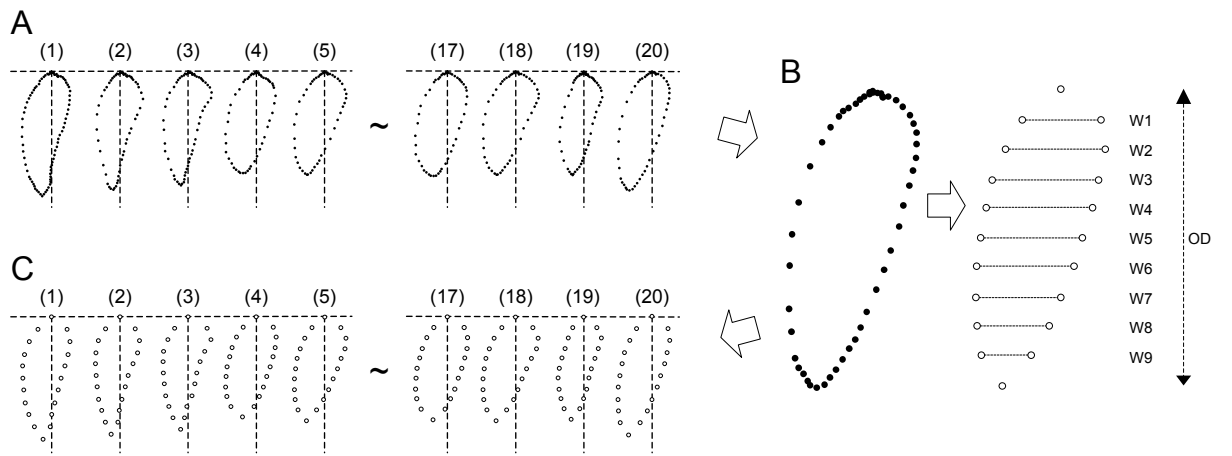

Fig. 1 Method used to calculate gape (opening distance), and masticatory width. A: Each cycle of the 1st-5th and the 17th-20th cycle. B: The chewing envelope was divided vertically into ten equally spaced sections, and the crossing of the movement path at each sectional crossing was determined (example of the twentieth cycle). OD: Opening distance, W1-W9: Masticatory width. C: Coordinates for each cycle were determined by vertical division into ten equally spaced sections.
Table 1 The numerical data of the masticatory width. Open: Lateral opening, Close: Lateral closing.

\begin{tabular}{cccc}
\hline Level & Open & Close & Width \\
\hline 1 & -1.9 & 1.8 & 3.8 \\
2 & -2.1 & 2.6 & 4.7 \\
3 & -1.8 & 3.2 & 5.0 \\
4 & -1.5 & 3.5 & 5.0 \\
5 & -1.0 & 3.8 & 4.8 \\
6 & -0.6 & 4.0 & 4.6 \\
7 & 0.0 & 4.0 & 4.0 \\
8 & 0.6 & 4.0 & 3.4 \\
9 & 1.4 & 3.8 & 2.4 \\
\hline Mean & & & 4.2 \\
\hline
\end{tabular}

intra-individual variations would be minimal during mastication of softened chewing gum. To this end, spatial and temporal parameters of the mandibular incisal point were quantitatively analyzed for 10 consecutive cycles of mastication in healthy subjects.

\section{Materials and Methods Subjects and test food}

Twenty healthy subjects (10 males and 10 females; mean age 28.6 years) between 21 and 44 years of age were selected. Informed consent was obtained, after the general nature of the study had been explained to the subjects. None of the subjects had clinical abnormalities in the masticatory system. The following selection criteria were applied: no complaints about their bite, a full complement of teeth excluding third molars, no major dental restorations, and no orthodontic treatment received. Softened chewing gum (Trident ${ }^{\circledR}$, Warner-Lambert, Parsippany, NJ, USA) was used as a test food. The weight was approximately $1 \mathrm{~g}$.

\section{Recording and analyzing method}

The subjects were seated comfortably in a chair so that their Frankfort horizontal plane was parallel to the floor. The movement of the mandibular incisal point during mastication of the test food for 20 seconds on the habitual chewing side was recorded by Mandibular Kinesiograph (MKG K-6I, Myotronics, Seattle, WA, USA).

For 20 cycles from the 1 st cycle to the 20 th cycle, the spatial parameters (gape and masticatory width) and temporal parameter (cycle time) were calculated (Fig. 1, Table 1), and the changes in these parameters were evaluated. Then the 20 cycles of mastication were classified into 11 series of 10 consecutive cycles; the 1st series being the 1 st to the 10 th cycles, the 2 nd series being the 2 nd to the 11th cycle, and so on. The coefficients of variation of the spatial and temporal parameters for each series were calculated and were compared among the series.

All the data were analyzed with statistical software (SPSS for Windows 10.0J, SPSS, Chicago, IL, USA). The changes in the spatial and temporal parameters were evaluated using repeated-measures analysis of variance. When significant effects were identified among the series by analysis of variance, Bonferroni's multiple comparison test was performed. All statistical analyses were performed with the significance set at the 0.05 and 0.01 probability levels.

\section{Results}

The gape, masticatory width, and cycle time were maximal at the first cycle of mastication. They decreased progressively until the fourth or fifth cycle, and remained almost unchanged thereafter until the 20th cycle (gape: $\mathrm{F}=8.02, p<0.01$; masticatory width: $\mathrm{F}=11.19, p<0.01$; cycle time: $\mathrm{F}=30.86$, $p<0.01)$. The coefficients of variation for the gape, masticatory width, and cycle time were maximal during the first series. They decreased progres- 

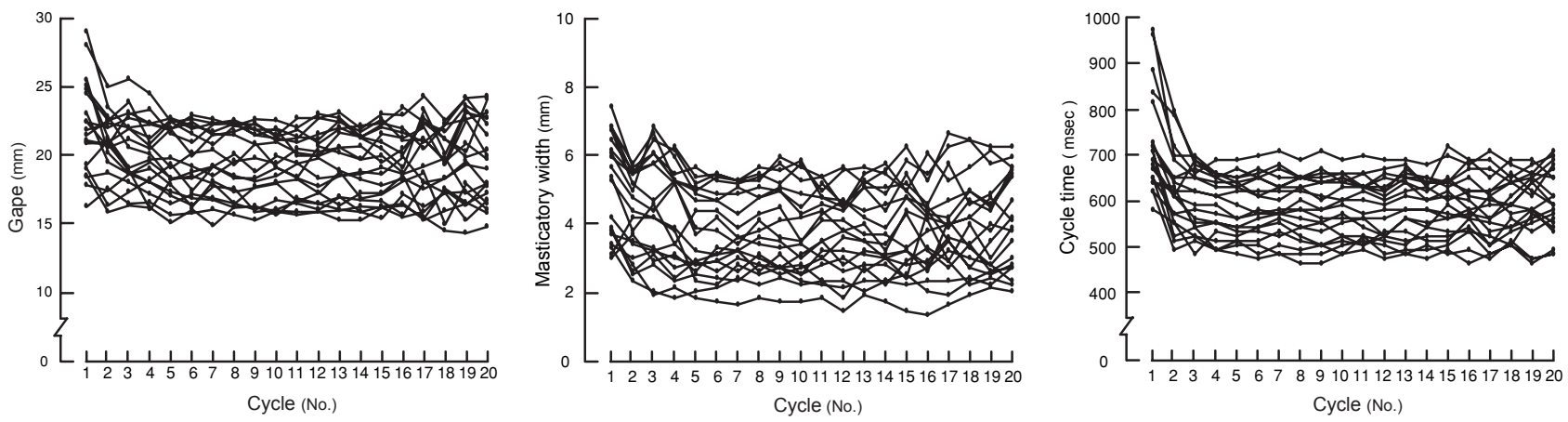

Fig. 2 The gape, masticatory width, cycle time of each cycle from the 1st cycle to the 20 th cycle for each subject.

Table 2 Coefficients of variation for the gape, masticatory width, and cycle time.

\begin{tabular}{lrrrrrrrrrrr}
\hline Series & 1 & 2 & 3 & 4 & 5 & 6 & 7 & 8 & 9 & 10 & 11 \\
\hline Gape & $6.7^{* *}$ & $5.1^{* *}$ & 4.3 & 3.7 & 3.2 & 3.2 & 3.5 & 3.7 & 4.1 & $4.5^{*}$ & $4.7^{* *}$ \\
Masticatory width & $15.4^{* *}$ & $11.5^{* *}$ & $11.2^{*}$ & 9.4 & 7.7 & 8.4 & 9.2 & 10.5 & 10.7 & $11.0^{*}$ & $11.5^{* *}$ \\
Cycle time & $7.9^{* *}$ & $4.8^{*}$ & 3.9 & 3.5 & 3.6 & 3.7 & 3.9 & 4.1 & 4.3 & 4.5 & $4.9^{*}$ \\
\hline & & & & & & & & & $*: p<0.05, * *: p<0.01$
\end{tabular}

sively until the fourth to sixth series, and tended to increase gradually thereafter. Minimal coefficients of variation were observed during the fifth and sixth series for the gape, during the fifth series for the width, and during the fourth series for the cycle time (Table 2). Significant differences were noted between series 5-6 and series 1, 2, 10, and also 11 in the gape, between series 5 and series $1-3,10$ and also 11 in the width, and between series 4 and series 1, 2, and also 11 in the cycle time.

\section{Discussion}

It is known that food becomes softer and less bulky during mastication, ${ }^{2,5,19}$ and that masticatory movements can vary depending on differences in the properties of the food. With regard to the changes in the initial masticatory movements after the start of mastication, Jemt et $\mathrm{al}^{20}$ investigated the gape and cycle time for the first 16 cycles of mastication of bread in healthy subjects. They reported that the cycle time became markedly shorter until the fourth or fifth cycle, and that it remained constant thereafter until the 12 th cycle, followed by a tendency toward decrease from the 12 th cycle onward. Similar findings have also been reported by some other investigators, including a report that the number of cycles needed until stabilization of masticatory movements tended to be greater for foods that are difficult to chew. ${ }^{2,21}$ In this study, softened chewing gum, that shows little change in size or hardness during mastication, was used as the test food. All of the parameters examined, namely, the gape, masticatory width, and cycle time were maximal during the first cycle and decreased progressively thereafter until the fourth or fifth cycle, followed by maintenance at an approximately constant level until the 20th cycle. These results were consistent with previous reports. Therefore it may be possible to say that irrespective of the properties of the food, masticatory movements are most pronounced during the first cycle and decrease progressively during the subsequent cycles, followed by maintenance at an approximately constant level. Furthermore, considering that several cycles were required until stabilization of the masticatory movements and that a greater number of cycles were needed until stabilization of mastication of foods that are difficult to chew, we may say that the first several cycles of mastication determine the nature of the subsequent masticatory movements.

For each of the consecutive 10 cycles (first to eleventh series) the coefficients of variation of the spatial and temporal parameters were minimal during the fourth to sixth series, and there were significant differences between series 4-6 (during which the values were minimal) and series $1-3$ and also 10-11. These results indicate that the mastica- 
tory movements varied between the initial cycles and the latter cycles of the 20-cycle period. On the other hand, the coefficients did not differ significantly between series $4-6$ and series $4-9$. This provides objective evidence for the view that masticatory movements remain almost constant for around a little over 10 cycles after the initial several cycles. The presence of such a constant section of masticatory movement is possibly attributable to the basic masticatory rhythm formed primarily by the pattern generator in the brain stem. ${ }^{1}$ This rhythm appears to allow mastication to be performed unconsciously in a stable manner, once the rhythm has been established. The finding of a change after the stable cycle period (a little over 10 cycles) may suggest that pooling of saliva causes changes in the feedback signals from the periphery over time, resulting in the regulation of masticatory movements by the central nervous system, which then plays a role in the regulation. Another possible view is that the unconsciously stable mastication can be continued for only around 10 cycles, and that conscious elements are added during the course of mastication, which result in feedback effects on the movements. Further studies would be useful to resolve these questions.

In this study, the masticatory movements during mastication of softened chewing gum stabilized over six series (fourth to ninth), during which the coefficients of variation of the spatial and temporal parameters showed no significant differences from those obtained in the series during which the coefficients were minimal. These series may be recommendable as sections of mastication showing minimal intra-individual variations. Among others, the fourth to sixth series, during which the co- $^{-}$ efficients of variation were minimal, may be viewed as optimal sections for the evaluation of masticatory movements during mastication of softened chewing gum.

\section{Conclusion}

The intra-individual variations in masticatory movement during mastication of softened chew ing gum are minimal during the fourth through the sixth series, i.e., during the ten cycles following the fourth to the sixth cycle of mastication.

\section{References}

1. Lund JP, Kolta A, Westberg KG et al. Brainstem mechanisms underlying feeding behaviors. Curr Opin Neurobiol 1998; 8: 718-724.

2. Schindler HJ, Stengel E, Spiess WE. Feedback control during mastication of solid food textures - a clinical-experimental study. J Prosthet Dent 1998; 80: 330-336.

3. Shiga H, Stohler CS, Kobayashi Y. The effect of bolus size on the chewing cycle in humans. Odontology $2001 ; 89: 49-53$.

4. Anderson K, Throckmorton GS, Buschang PH et al. The effects of bolus hardness on masticatory kinematics. J Oral Rehabil 2002; 29: 689-696.

5. Bhatka R, Throckmorton GS, Wintergerst AM et al. Bolus size and unilateral chewing cycle kinematics. Arch Oral Biol 2004; 49: 559-566.

6. Mongini F, Tempia-Valenta G. A graphic and statistical analysis of the chewing movements in function and dysfunction. J Craniomandibular Pract 1984; 2: 125-134.

7. Kuwahara T, Bessette RW, Maruyama T. Chewing pattern analysis in TMD patients with and without internal derangement: Part I. Cranio 1995; 13: 8-14.

8. Martín C, Alarcón JA, Palma JC. Kinesiographic study of the mandible in young patients with unilateral posterior crossbite. Am J Orthod Dentofacial Orthop 2000; 118: 541-548.

9. Neto GP, Puppin-Rontani RM, Garcia RC. Changes in the masticatory cycle after treatment of posterior crossbite in children aged 4 to 5 years. Am J Orthod Dentofacial Orthop 2007; 131: 464-472.

10. Rilo B, da Silva JL, Mora MJ et al. Unilateral posterior crossbite and mastication. Arch Oral Biol 2007; 52: 474-478.

11. Foster KD, Woda A, Peyron MA. Effect of texture of plastic and elastic model foods on the parameters of mastication. J Neurophysiol 2006; 95: 3469-3479.

12. Jemt T, Olsson K. Computer-based analysis of the single chewing cycle during mastication in repeated registrations. J Prosthet Dent 1984; 52: 437-443.

13. Svensson $\mathrm{P}$, Arendt-Nielsen L, Bjerring $\mathrm{P}$ et al. $\mathrm{Hu}^{-}$ man mastication modulated by experimental trigeminal and extra-trigeminal painful stimuli. $\mathrm{J}$ Oral Rehabil 1996; 23: 838-848.

14. Drewing K, Aschersleben G. Reduced timing variability during bimanual coupling: a role for sensory information. Q J Exp Psychol A 2003; 56: 329-350.

15. Sato S, Kawamura H. Natural course of non-reducing disc displacement of the temporomandibular joint: changes in electromyographic activity during chewing movement. J Oral Rehabil 2005; 32: 159165.

16. Wintergerst AM, Buschang PH, Hutchins B et al. Effect of an auditory cue on chewing cycle kinemat- 
ics. Arch Oral Biol 2006; 51: 50-57.

17. Shiga H, Kobayashi Y, Arakawa I et al. Selection of food and chewing side for evaluating masticatory path stability. Odontology 2003; 91: 26-30.

18. Shiga H, Kobayashi Y. An objective evaluation of masticatory function by analysis of masticatory movements. J Jpn Prosthodont Soc 1990; 34: 11121126.

19. Blissett A, Prinz JF, Wulfert F et al. Effect of bolus size on chewing, swallowing, oral soft tissue and tongue movement. J Oral Rehabil 2007; 34: 572-582.

20. Jemt T, Hedegård B. Reproducibility of chewing rhythm and of mandibular displacements during chewing. J Oral Rehabil 1982; 9: 531-537.

21. Inaba J, Shiga H, Kobayashi Y. Peripheral feedback adjustment in chewing various types of food. J Jpn Prosthodont Soc 2001; 45: 271-282. 